\title{
Illuminating the functional and structural repertoire of human TBC/RABGAPs
}

Marieke A. M. Frasa, Katja T. Koessmeier, M. Reza Ahmadian and Vania M. M. Braga

Nature Reviews Molecular Cell Biology 13, 67-73 (2012)

Since the publication of this article, the authors have further optimized the sequence analysis of the RABGAP TBC domains in Supplementary information S1 (figure), using more stringent criteria that also include the homology of the sequences surrounding the catalytic fingers. This has allowed them to identify a consensus sequence motif and to redefine the proposed grouping of TBC/RABGAPs in BOX 1 and Supplementary information S2 (table). A revised version of BOX 1 is shown below.

\footnotetext{
Box 1 | Classical and unconventional TBC/RABGAPs

The presence of a dual-finger mechanism is, to our current knowledge, a unique feature of the Tre2-Bub2-Cdc16 (TBC) domain, which uses two catalytic residues, $R$ and $Q$, to stabilize the transition state and catalyse the GTP hydrolysis of RABs ${ }^{72}$. Analysis of TBC domain-containing RAB-specific GTPase-activating proteins (TBC/RABGAPs) revealed that they predominantly share six conserved regions (A-F). A consensus sequence motif is deduced from the conserved regions $B$ and $C$ (Supplementary information S1 (figure)): it emphasizes two distinct signatures containing $R$ and $Q$ fingers found in 29 family members. These proteins are known as classical TBC/RABGAPs (group 1: RQ; see table). Interestingly, 13 unconventional TBC/RABGAPs lacking the $\mathrm{R}$ and/or $\mathrm{Q}$ fingers have been identified (see below). If these unconventional TBC/RABGAPs do inactivate RABs, they probably use a different mode of action.

We propose to group these unconventional TBC/RABGAPs into four groups according to the deviations of the catalytic fingers (see the table). The first three proposed groups comprise: TBC1 domain family members 29 and 13 (TBC1D29 and TBC1D13; group 2: RX), transferrin receptor-like 2 (TRE2; group 3: RR), TBC1D3 and TBC1D26 (group 4: XR). These proteins share conserved regions within the TBC domains with the classical TBC/RABGAPs (see Supplementary information S1 (figure)). By contrast, the members of the last proposed group of TBC/RABGAPs lack the RQ finger (group 5: XX) and show poor sequence similarity to the classical TBC domains, especially within the conserved regions $B$ and $C$. Furthermore, a few proteins have incomplete TBC domains .

With two exceptions, no substrate has yet been reported for these unconventional TBC/RABGAPs (see Supplementary information S2 (table)). TBC1D3 (group 4: XR) undergoes a persistent interaction with RAB5 and is thus thought to be an effector of this RAB ${ }^{51}$. Taken together, the homology within the $T B C$ regions $(A-F)$ and the fact that $R Q$ fingers are indispensable catalytic residues ${ }^{36}$ suggest that the proteins in groups $R X, R R, X R$ and $X X$ may be inactive TBC/RABGAPs. Interestingly, out of the eight proteins in group XX, TBC1D7 has been reported to be a GAP for RAB17 (REF. 8), indicating that this group might work through an alternative mechanism to stimulate GTP hydrolysis. Nevertheless, an inactive TBC domain may still have important cellular functions to coordinate different signalling pathways, as is observed with TBC1D3 and TRE2 (see main text) and, similarly, with the RHOGAP-like protein OCRL1, which has no R finger but interacts with RAB8 (REF. 73).
}

EVI5, ecotropic viral integration site 5; RN-TRE, related to the N terminus of TRE; SGSM, small G protein signalling modulator.

\section{SUPPLEMENTARY INFORMATION} See online article: $\underline{\mathrm{S} 1}$ (figure) $\mid \underline{\mathrm{S} 2}$ (table) ALL LINKS ARE ACTIVE IN THE ONLINE PDF 